\title{
A dual-genome microarray for the pea aphid, Acyrthosiphon pisum, and its obligate bacterial symbiont, Buchnera aphidicola Alex CC Wilson*1, Helen E Dunbar ${ }^{1}$, Gregory K Davis ${ }^{2}$, Wayne B Hunter ${ }^{3}$, David L Stern ${ }^{2}$ and Nancy A Moran ${ }^{1}$
}

\author{
Address: ${ }^{1}$ Department of Ecology and Evolutionary Biology, University of Arizona, Tucson, AZ, 85721, USA, ${ }^{2}$ Department of Ecology and \\ Evolutionary Biology, Princeton University, Princeton, NJ, 08544, USA and ${ }^{3}$ United States Department of Agriculture, Agricultural Research \\ Service, U.S. Horticultural Research Laboratory, Fort Pierce, FL, 34945, USA \\ Email: Alex CC Wilson* - acwilson@email.arizona.edu; Helen E Dunbar - dunbar@email.arizona.edu; \\ Gregory K Davis - gkdavis@Princeton.EDU; Wayne B Hunter - whunter@ushrl.ars.usda.gov; David L Stern - dstern@Princeton.EDU; \\ Nancy A Moran - nmoran@u.arizona.edu \\ * Corresponding author
}

Published: I4 March 2006

BMC Genomics2006, 7:50 doi:10.1186/147/-2164-7-50
Received: 26 September 2005

Accepted: 14 March 2006

This article is available from: http://www.biomedcentral.com/147I-2/64/7/50

(C) 2006Wilson et al; licensee BioMed Central Ltd.

This is an Open Access article distributed under the terms of the Creative Commons Attribution License (http://creativecommons.org/licenses/by/2.0), which permits unrestricted use, distribution, and reproduction in any medium, provided the original work is properly cited.

\begin{abstract}
Background: The best studied insect-symbiont system is that of aphids and their primary bacterial endosymbiont Buchnera aphidicola. Buchnera inhabits specialized host cells called bacteriocytes, provides nutrients to the aphid and has co-speciated with its aphid hosts for the past 150 million years. We have used a single microarray to examine gene expression in the pea aphid, Acyrthosiphon pisum, and its resident Buchnera. Very little is known of gene expression in aphids, few studies have examined gene expression in Buchnera, and no study has examined simultaneously the expression profiles of a host and its symbiont. Expression profiling of aphids, in studies such as this, will be critical for assigning newly discovered $A$. pisum genes to functional roles. In particular, because aphids possess many genes that are absent from Drosophila and other holometabolous insect taxa, aphid genome annotation efforts cannot rely entirely on homology to the best-studied insect systems. Development of this dual-genome array represents a first attempt to characterize gene expression in this emerging model system.

Results: We chose to examine heat shock response because it has been well characterized both in Buchnera and in other insect species. Our results from the Buchnera of $A$. pisum show responses for the same gene set as an earlier study of heat shock response in Buchnera for the host aphid Schizaphis graminum. Additionally, analyses of aphid transcripts showed the expected response for homologs of known heat shock genes as well as responses for several genes with unknown functional roles.

Conclusion: We examined gene expression under heat shock of an insect and its bacterial symbiont in a single assay using a dual-genome microarray. Further, our results indicate that microarrays are a useful tool for inferring functional roles of genes in A. pisum and other insects and suggest that the pea aphid genome may contain many gene paralogs that are differentially regulated.
\end{abstract}



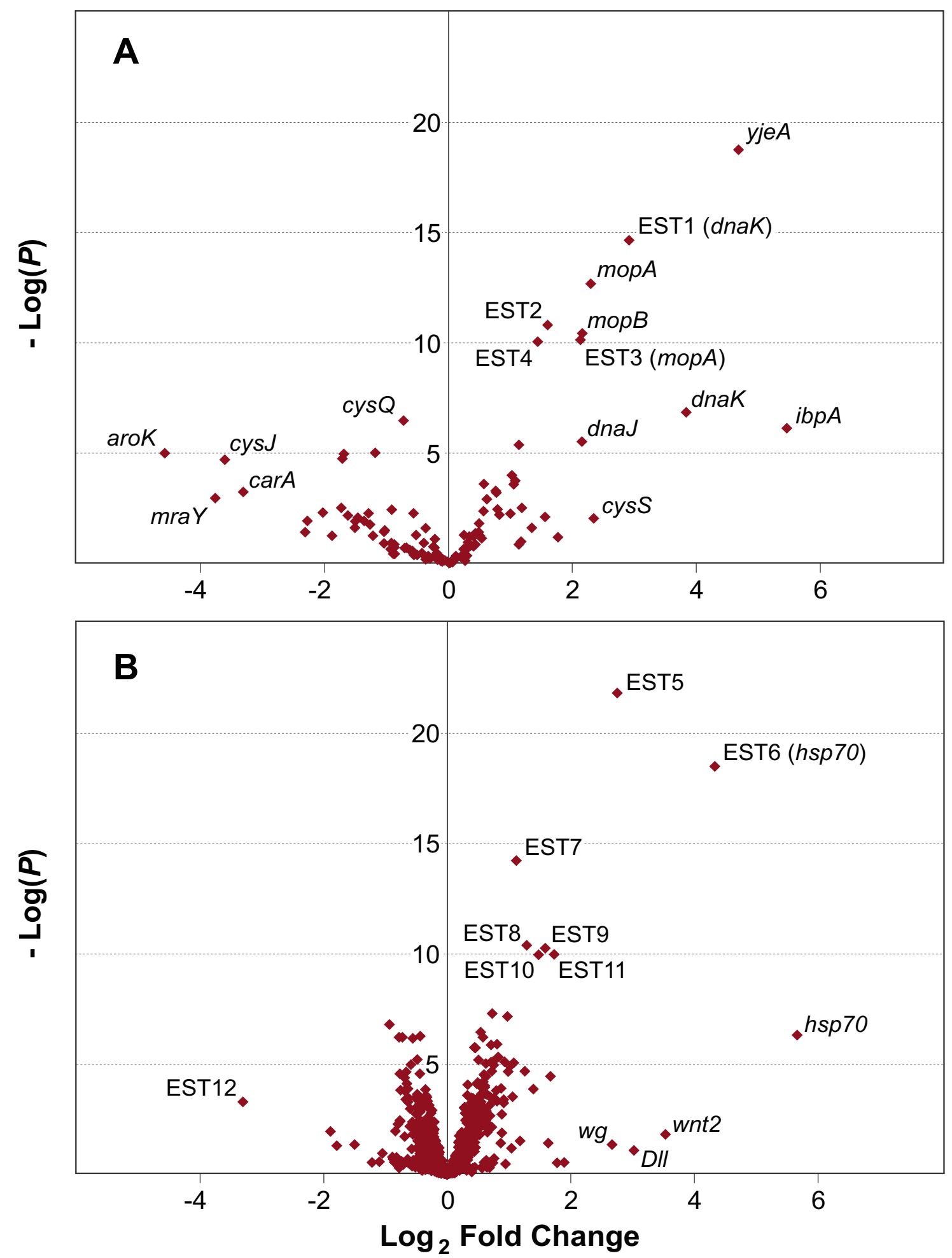

Figure I

Fold-change in gene expression after exposure to heat stress. Line at $\log (P)=5$ corresponds to $P=0.000 \mathrm{I}$. A. Buchnera ESTI $($ dnaK $)=$ CN582666, EST2 (flil) $=$ CN5875 I 7, EST3 $($ mopA $)=$ CN585306, EST4 $(m e t E)=$ CN582733. B. Pea aphid, A. pisum. EST5 $=$ CN584I39, EST6 $($ hsp70) $=$ CN586725, EST7 $=$ CF588076, EST8 $=$ CN586853, EST9 $=$ CN585958, ESTI0 $=$ CF587727, ESTII = CN587624, ESTI $2=$ CN584928. 


\section{Background}

Ancient and now highly specialized associations with bacterial symbionts are widespread in invertebrate groups that feed on nutrient-poor diets [1]. Such associations are common in insects, in which the best studied symbiotic system is that of aphids (Insecta: Hemiptera: Aphididae) and their primary symbiont Buchnera aphidicola [2]. Buchnera, which has not been established in laboratory cultures, inhabits specialized host cells (bacteriocytes), is required for nutrition (host development, growth and reproduction) and has been strictly vertically transmitted from mother to offspring for the last 150 million years [25]. In addition to being the best studied insect-symbiont system [6], aphids are of general interest due to their economic and agricultural status as plant pests and plant disease vectors [7] and their complex life cycles with multiple developmental pathways and parthenogenesis $[8,9]$.

The pea aphid Acyrthosiphon pisum (Insecta: Hemiptera: Aphididae) will be one of the first insect species outside of the Holometabola to serve as a model system in genomics [10]. Currently, little is known about gene expression in this newly emerging model organism. In addition, it is already clear from the growing collection of aphid ESTs that aphids possess many genes that are absent from Drosophila and other holometabolous insect taxa [11-13]. Accurate and successful interpretation of the A. pisum genome sequence will depend critically on studies of gene expression under different environmental conditions, in different tissues and morphs, and at different developmental stages $[14,15]$.

The complete genome sequences are now available for the Buchnera from three aphid species $[5,16,17]$, including $A$. pisum [5]. Facilitated by these genome sequences and other studies, Buchnera has become a model for studying the evolution of symbiotic and pathogenic lifestyles in bacteria $[6,18,19]$.

In the present study we take a metagenomic approach [20] to examining transcriptional responses to heat shock in a host/symbiont system, using pooled RNA samples and a microarray containing gene sets from both organisms. Select results were verified with reverse-transcriptase quantitative PCR (RT-qPCR). To our knowledge, this dual-genome microarray is the first used to examine simultaneously the pooled transcriptome of an animal host and its symbiont; a previous dual-genome array has been used to study Rhizobium-plant interactions [21].

The transcriptional response to heat shock has been well characterized in other Buchnera [22] and in other insects [23], enabling us to generate $a$ priori predictions and thus to assess the success of our array design. Further, aphids are highly sensitive to heat, with considerable variation among species (e.g., [24-27]). Part of this sensitivity stems from the intolerance of the resident symbionts to high temperature. Aphid lineages can be rendered infertile as a consequence of heat-induced elimination of their resident Buchnera [28], and field populations of A. pisum exposed to naturally occurring high temperatures undergo a decline in the number of bacteriocytes, reflecting the decline of the Buchnera population within heat-stressed aphid hosts [29]. Thus, the overall thermal tolerance of this symbiotic system is dependent on both host and symbiont responses to thermal challenge.

To date, there are no published studies on heat shock responses of any aphid genes. Previously, Wilcox et al. [22] studied gene expression under heat shock in Buchnera derived from the aphid Schizaphis graminum and identified a set of nine genes that were significantly upregulated. Six of the nine genes, mopA (= groEL), mopB (= groES), dnaJ, dnaK, grpE, and $i b p A$ are heat shock genes in other bacteria such as the related species Escherichia coli and are known to function in the recycling and refolding of degraded proteins. Two additional genes, $f p r$ and the pseudogene of $y j e A$, showed elevated transcript levels following heat shock. Both are positioned immediately downstream of $i p b A$ and its $\sigma^{32}$ heat shock promoter, and their response reflects transcriptional read-through based on reverse transcriptase quantitative PCR assays of different regions of the transcripts [22].

\section{Results and discussion}

\section{Heat shock response in Buchnera of A. pisum}

Overall, the set of genes responding to heat shock in the Buchnera of A. pisum is similar to the set previously identified in the Buchnera of S. graminum [22]. In both, the following genes belonging to the E. coli heat shock (HS) regulon were significantly upregulated: dnaK, торA (= groEL), mopB (= groES), ibpA and $d n a J$ (Figure $1 \mathrm{~A}$ ). (We also detected a similar fold-change in expression of the $E$. coli HS regulon gene, grpE, in Buchnera of A. pisum as reported for Buchnera of $S$. graminum, but this change was not statistically significant.) In Buchnera of both aphid species, yjeA is downstream of $i b p A$ and $f p r$, but encoded on the opposite strand. Our results show an increase in yjeA transcript with heat shock in Buchnera of A. pisum, as described for Buchnera of $S$. graminum. Because the microarray probes consist of double-stranded amplicons, the most likely interpretation is that the heat-shock induced transcription of ibpA continues through both $f p r$ and yjeA, resulting in elevated transcription of the non-coding strand of $y j e A$ (the gene fpr is not present on our array). We verified this explanation for $y j e A$ transcription under HS through targeted RT-PCR of a region overlapping $i b p A$, fpr, and the complement of yjeA (data not presented), using the same approach applied to Buchnera of S. graminum [22]. 


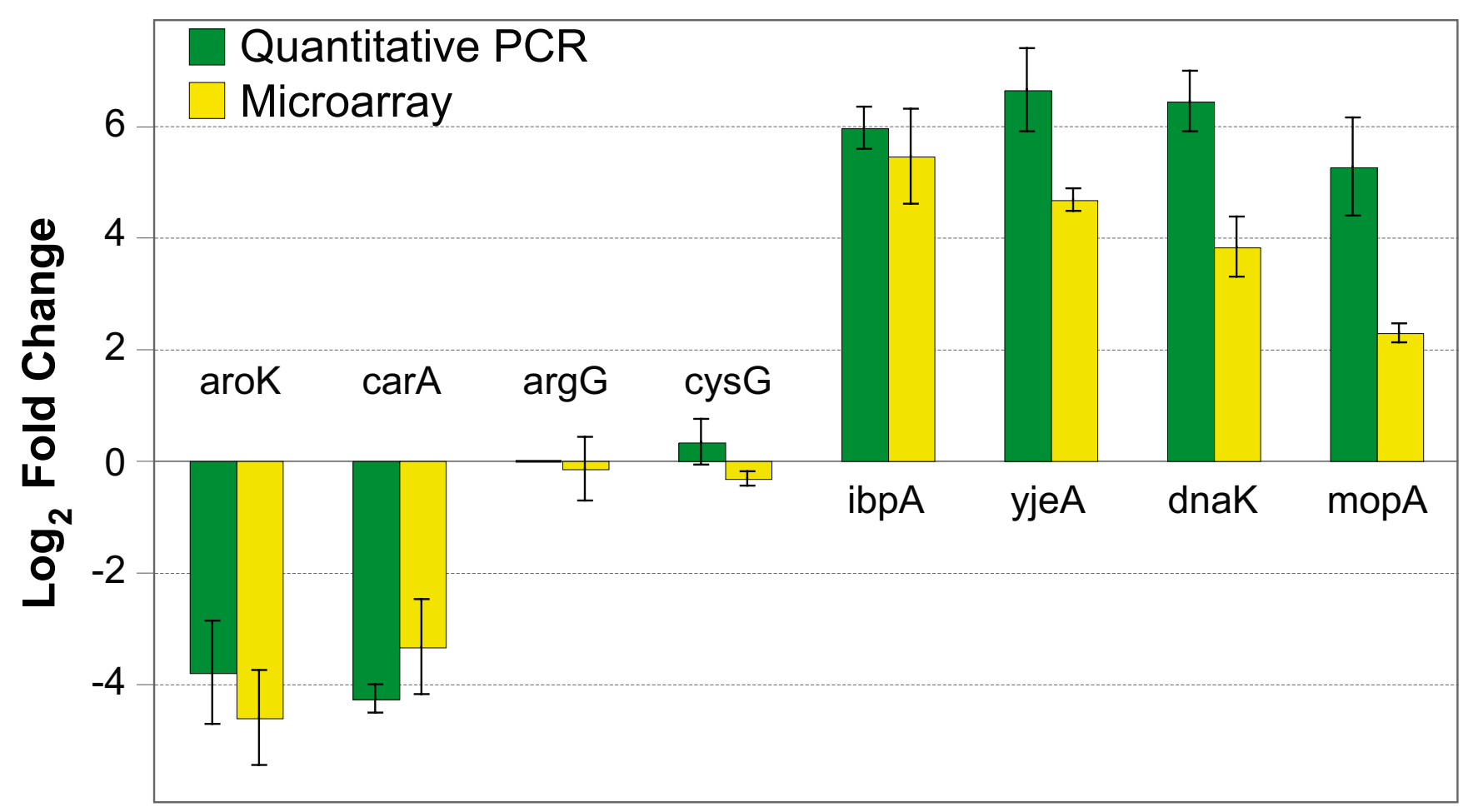

Figure 2

Mean fold-change estimates for select Buchnera of $A$. pisum genes compared between microarray and RTqPCR methods. Standard error bars are shown. RT-qPCR data have been standardized relative to argG.

In addition to the heat-responsive genes identified for Buchnera of S. graminum [22], two other Buchnera probes showed increased signal following HS (Figure 1A): EST2 (fliI), flagellum-specific ATP synthase and EST4 (metE), 5methyltetrahydropteroyltriglutamate-homocysteine Smethyltransferase. The gene metE was present as two types of probe: one, EST4, showed consistent increase in signal under HS, and the other, a directly amplified product from genomic DNA, did not respond. Our interpretation is that the spot corresponding to EST4 was contaminated with some other sequence, and that metE is not responding.

Signal from several Buchnera genes was decreased following HS (Figure 1A). These include aroK, mraY, cysJ, carA and $c y s Q$. Of these, carA also showed down-regulation under HS in the study of Buchnera of S. graminum [22].

Using RT-qPCR, to investigate the accuracy of our microarray results for a select set of eight Buchnera genes, we found good congruence between methods, using the nonHS genes $\arg G$ and $c y s G$ as controls (Figure 2, data for $c y s G$ not shown).

While the set of genes responding under HS corresponded to the set identified for Buchnera of S. graminum, the mag- nitude of response was much larger for Buchnera of $A$. pisum, both from the microarray and the RT-qPCR estimates. The HS treatment was the same for both studies; yet, expression changes ranged from under 2-fold to 13fold in Buchnera of S. graminum [22] compared with 5fold to 70-fold in Buchnera of A. pisum (Figure 1A, Figure 2).

\section{Heat shock response in A. pisum}

Two paralogs of the HS gene, hsp70, were included on the array, one derived from a PCR product amplified from genomic DNA (hsp70) and the other derived from an EST (Figure 1B, EST6 - CN586725). Both showed greater than 16-fold increase in transcript abundance under HS (Figure 1B). Seven other genes (Figure 1B, EST5 and EST7 EST12) showed large fold-changes and/or statistically significant changes in gene expression under HS conditions.

We investigated the accuracy of our microarray results using RT-qPCR for eight $A$. pisum genes representing a range of responses on the array. Two of these showed no change on the array and were used as control genes (EST13 (CN587115, a homolog of EF1a) and EST14 (CN582657)), and both control genes gave similar results. We found good congruence between microarray and RT-qPCR methods for aphid EST 6 (a homolog of 
Table I: Spike microarray hybridization data listing genes showing significant signal increase. Genes that were used to spike the control RNA are marked * for Buchnera genes and ** for the aphid gene. Positive $\log _{2}$ fold-changes indicate genes that cross-hybridized with aphid $h s p 70$ and negative $\log _{2}$ fold-changes indicate genes that cross-hybridized with the Buchnera genes. Genes reported are those that had $\log _{2}$ fold-changes $\geq|I|$ and $a-\log (P) \geq 2$. Genes are listed in order of signal strength.

\begin{tabular}{|c|c|c|c|}
\hline Gene & $\log _{2}$ fold-change & Standard Error & $-\log (P)$ \\
\hline hsp70** & 7.66 & 0.29 & 11.90 \\
\hline EST6 - hsp70 & 4.26 & 0.29 & 8.82 \\
\hline DII & 2.55 & 0.37 & 4.98 \\
\hline wg & 2.06 & 0.44 & 3.36 \\
\hline EST I5 - hsp70/hsp68 & 1.84 & 0.33 & 4.00 \\
\hline EST5 & 1.28 & 0.21 & 4.35 \\
\hline wnt2 & 1.23 & 0.34 & 2.46 \\
\hline CN585728 & -1.14 & 0.16 & 5.04 \\
\hline EST7 & -1.51 & 0.33 & 3.28 \\
\hline EST3 - mopA & -1.57 & 0.34 & 3.29 \\
\hline CN582302 & -1.71 & 0.30 & 4.13 \\
\hline$m o p A^{*}$ & -2.29 & 0.45 & 3.79 \\
\hline ESTI - dnaK & -4.04 & 0.39 & 6.99 \\
\hline$d n a K^{*}$ & -5.48 & 0.43 & 7.97 \\
\hline$i b p A^{*}$ & -5.95 & 0.65 & 6.30 \\
\hline yjeA* & -7.13 & 0.17 & 14.58 \\
\hline
\end{tabular}

hsp70) ( $\log _{2}$ fold-change estimate from microarray data: $5.67 \pm$ S.E. 0.87 c.f. $\log _{2}$ fold-change estimate from RTqPCR: $7.32 \pm$ S.E. 1.03). However, RT-qPCR indicated no significant change in relative expression for five of the aphid ESTs that showed either upregulation (EST5, EST7, EST8 and EST10) or down-regulation (EST12) under HS. This indicates that expression of the designated gene (corresponding to the EST and to the sequence matching the PCR primers), does not change under HS. Two explanations are (1) that a paralogous gene is undergoing change in expression giving cross-hybridization on the array but not giving PCR product because of the greater stringency of the RT-qPCR and (2) that there is contamination of the arrayed spots attributable to splash during transfers to the microtiter plates or carry-over of the array pins.

To discriminate between these two possibilities, we performed a dye-flip microarray hybridization experiment using samples differing only in the presence or absence of added amplicons of known HS genes represented on the array. RNA representing the transcript pool of aphids under control conditions was divided into two portions, one spiked with amplicons of four upregulated Buchnera genes (dnaK, ibpA, mopA, yjeA) and one spiked with the amplicon of an upregulated aphid gene ( $h s p 70$ ). Two of the five aphid genes that showed incongruent results (EST8 and EST10) did not change expression in the spike experiment. This indicates that the observed microarray results from the HS experiment cannot be explained as the result of contamination among probes on the array.
Instead, they likely reflect cross-hybridization of closely related gene transcripts [30].

Non-target sequences with $>70 \%$ sequence identity can cross-hybridize to cDNA probes [31]. This is illustrated on our array, which contains three members of the aphid $h s p 70$ gene family; $h s p 70$, which was amplified using PCR primers from genomic DNA for inclusion on this array and for which we have a cloned PCR product; EST6, which was isolated from EST libraries (Figure 1) and EST15 (CN584562), which shows homology to hsp70 and hsp68 from other insect taxa and did not show significant upregulation under HS conditions (Table 1). PCR primers designed to EST6 do not amplify our cloned $h s p 70$ product or EST15; however, in the spike experiment, $h s p 70$ PCR product hybridized to itself and to both the EST6 and EST15 probes (Table 1). Similarly, expression changes in paralogous genes are the most evident explanation for the change in signal of EST8 and EST10 (Table 1). The final two aphid ESTs, EST5 and EST7, did show significant "upregulation" in the spiked pools (Table 1). The EST5 probe cross-hybridized with $h s p 70$ and the EST7 probe with one of the four Buchnera genes. We could not find any significant stretches of sequence similarity to explain these results, suggesting that these probes were contaminated. The EST5 and EST7 results are most readily attributable to splash on the microtiter plates. However, results from the spike experiment (Table 1) clearly indicate that apparent upregulation of $w g$, wnt 2 and $\mathrm{Dll}$ (fold-changes of 6-12 under HS, without strong statistical significance 
(Figure 1B)) resulted from pin carryover of the $h s p 70$ probe during the printing process. The same pin stamped $h s p 70, D l l, w g$ and $w n t 2$, in that order.

\section{Usefulness of EST-based microarrays for functional annotation of the aphid genome}

These results suggest that, in addition to $h s p 70$, paralogs of EST8 and of EST10 undergo significant upregulation under HS. Both have homologs in many other taxa, but we found no reports of involvement in the HS response in other organisms. EST8 shows significant homology to tyrosyl-tRNA synthase from Homo sapiens, Mus musculus and Drosophila virilis, while EST10 is homologous to the gene encoding the molybdenum cofactor of sulfurase in Bombyx mori, Bos taurus and Homo sapiens. Molybdenum cofactor sulfurase is responsible for xanthine dehydrogenase activation in various organisms, a gene which plays a critical role in nitrogen metabolism, especially in insects [32]. Our results suggest that genes that share significant sequence similarity to these two ESTs have a function in the heat stress response in aphids.

\section{Conclusion}

One of the outstanding questions in host/symbiont systems is how gene expression in the partner organisms is coordinated. EST studies of aphid bacteriocytes have shown elevated expression of genes associated with a range of cellular functions including amino acid metabolism, cell transport, and antibacterial activity [13]. The presence of these functional categories at high frequency in bacteriocyte EST libraries highlights important aspects of genome coordination between symbiont and host. Further progress in understanding how and when these genes are upregulated and the roles they play in mediating the symbiosis will require extensive expression studies using arrays such as the one we describe here.

We have demonstrated the feasibility of using a dualgenome microarray to simultaneously study the expression profile of an aphid and its obligate bacterial symbiont. The success of this approach is dependent on the relative abundances of transcripts from the partner organisms. Buchnera occurs in large numbers within aphids; their genomes constitute about 5-10\% of total DNA content [3]. A quantitative PCR study of gene copy number in intact adult pea aphids indicated a ratio of approximately 7.5 single copy Buchnera genes for each single copy aphid gene (H. Dunbar and N. Moran, unpublished data). This estimate is consistent with previous estimates of genome sizes of the two organisms and of the proportion of total DNA belonging to Buchnera [3]. Thus, we expected transcript abundances of Buchnera and aphid genes to be comparable, enabling hybridization of the pooled sample on the same array without loss of signal for either organism. Use of a dual-genome array for the study of symbiosis in systems where symbionts are found in low copy number, e.g. Wolbachia, the facultative bacterial endosymbiont of Drosophila and other insects [33], is likely to prove more difficult. However, many animals, including many other insects and marine invertebrates, do possess large numbers of obligate primary symbionts. In these composite organisms, an understanding of the integration of component genomes requires the synchronous examination of gene expression in each member.

Finally, our results for the pea aphid genes suggest that the pea aphid genome may contain many gene paralogs that are differentially regulated. Preliminary analysis from an EST project suggests that the pea aphid genome may contain many gene duplications [34]. This fact will complicate analysis of differential gene expression in pea aphids using cDNA microarrays, and suggests that establishing the differential regulation of specific genes will require qPCR. The full extent of gene paralogy in the pea aphid will soon be revealed by whole genome sequencing [10].

\section{Methods}

Microarray design

We designed a 7,448 spot, dual-genome microarray containing 1,862 genes. The array includes 76 Buchnera genes representing the E. coli heat shock (HS) regulon (clpX, dnaJ, dnaK, grpE, hflB, hslU, hslV, htpX, htrA, ibpA, mopA and $m o p B$ ), essential amino acid biosynthesis genes (argA, $\arg B, \arg C, \arg D, \arg E, \arg F, \arg G, \arg H, \operatorname{aro} A, \operatorname{aroB}, \operatorname{aro} C$, aroD, aroE, aroH, aroK, carA, carB, cysD, cysG, cysH, cysI, $c y s J, c y s K, c y s N, c y s Q, d a p A, d a p B, \operatorname{dapD}$, dapE, dapF, glyA, hisB, hisC, hisD, hisF, hisI, ilvC, ilvH, ilvI, leuA, leuD, lysA, $m e t E$, pheA, thrA, thrB, $\operatorname{trp} A, \operatorname{trp} C$ and $\operatorname{trp} G$ ) and a number of other genes representative of various functional categories such as cold shock, cell wall biosynthesis and DNA repair (cspC, cysS, endA, lgt, mraY, mrcB, mrsA, murC, murE, murF, nlpD, prfB, serC, ung and yjeA). The array also includes, nine aphid developmental and heat shock genes (distal-less, eyeless, fused, nanos, vasa, vasa-like, wingless, wnt2 and hsp70) and 1777 A. pisum ESTs (GEO Accession: GSE3742). All Buchnera genes were amplified using genespecific primers designed from the Buchnera of A. pisum genomic sequence (GenBank Accession: BA000003, [6]). Primers were designed, where gene length permitted, to give an optimal product of 1000 base pairs with a melting temperature of $55^{\circ} \mathrm{C}$. If gene coding sequences were less than 1000 base pairs, primers were designed to maximize product length. We amplified Buchnera genes in two $50 \mu \mathrm{l}$ PCR reactions from a laboratory line of A. pisum. This line, 7-2-1 was collected in August 2001 in Cayuga County, New York from alfalfa by Jacob Russell. Since its collection, 7-2-1 has been maintained in continuous parthenogenetic culture in the laboratory of NAM. 
All A. pisum ESTs were derived from a cDNA library constructed in the laboratory of DLS (described in [34], GenBank Accessions: CF587442 - CF588411 and CN582088 CN587684). ESTs were clustered in a unigene set and 1824 genes were selected for inclusion based primarily on significant tblastx scores to other eukaryotes when used as queries in searches of the GenBank nr database. The corresponding cDNAs were amplified using $\mathrm{T} 3$ and $\mathrm{T} 7$ primers to pBluescript.

Genes were arranged in duplicate spots on slides, with Buchnera genes grouped into the first and second of 24 subarrays. In addition to spot duplication, the entire set was printed twice on each slide thus; each spot was printed in quadruplicate.

\section{Heat shock}

A single isofemale line of A. pisum was used in all HS experiments. This clone, called "TUC", was collected by NAM from fava bean in a garden in Tucson, Arizona in June 1999 and has been maintained in continuous parthenogenetic culture on fava bean at $20^{\circ} \mathrm{C}$ in the laboratory of NAM since its collection. Clone TUC, in addition to harboring the primary aphid symbiont, Buchnera, is host to the secondary bacterial symbiont, Candidatus Serratia symbiotica, previously known as the "R-type" [35].

Six to ten adult $A$. pisum were placed on a single fava bean plant in a cup cage and allowed to reproduce at $20^{\circ} \mathrm{C} 16$ L:8 D. After $24-48$ hours the adult aphids were removed from plants and flash-frozen in liquid nitrogen. First generation aphids were then maintained for one week, the developmental period required to attain the adult stage. Heat shock experiments were carried out $1-2$ days following ecdysis to adulthood. Prior to HS, 10 - 20 control aphids were removed from each plant, flash-frozen in liquid nitrogen and stored at $-80^{\circ} \mathrm{C}$ until RNA extraction and microarray hybridization. Heat shock conditions were applied by ramping from $20^{\circ} \mathrm{C}$ to $36^{\circ} \mathrm{C}$ over a two hour period at a rate of $2^{\circ} \mathrm{C} / 15 \mathrm{~min}$ and then maintaining aphids at $36^{\circ} \mathrm{C}$ for an additional 2 hours (4 hours in total). At 4 hours cultures were removed from the growth chamber and aphids were quickly collected into Eppendorf tubes, flash-frozen in liquid nitrogen and stored at $80^{\circ} \mathrm{C}$ until processing. Two biological replicates of clone TUC were exposed to heat shock in parallel.

\section{RNA extraction and microarray hybridization}

We extracted total RNA from control and HS samples using an RNeasy Mini Kit (Qiagen Cat. No.74104). RNA quantity and quality was assessed using an RNA LabChip ${ }^{\circledast}$ on the Agilent 2100 Bioanalyzer. We constructed cDNA from $10 \mu \mathrm{g}$ of total RNA using Omniscript Reverse Transcriptase and reaction buffer (Qiagen), 6 ng random hexamer primers, $0.5 \mathrm{mM}$ each dATP, dCTP, dGTP, $0.3 \mathrm{mM}$
dTTP, and $0.2 \mathrm{mM}$ amino-allyl linked dUTP. RNA was hydrolyzed, and purified cDNA populations were coupled to $\mathrm{Cy} 3$ or Cy5 fluors (Amersham). We flipped dye labels between treatments across experimental replicates. Therefore, results from 4 total slides were analyzed. Purified, labeled cDNA populations were suspended in $80 \mu \mathrm{l}$ Sigma Hybridization Buffer with $20 \mu \mathrm{g}$ Human COT1 DNA. Microarray slides were rehydrated, UV crosslinked, and denatured immediately before hybridization. Target samples were combined, denatured for $10 \mathrm{~min}$ at $94^{\circ} \mathrm{C}$, and pipetted into sealed, microvolume slide manifolds. We ran hybridizations in a GeneTAC automated station at $47^{\circ} \mathrm{C}$ for 16 hours and programmed 3 washes of variablestringency SDS/SSC solutions (Sigma).

\section{Analysis}

Signal intensities were measured using spot-finding software, softWoRx Tracker (version 2.20, MolecularWare Inc). Raw median spot intensity and median background intensity data were exported; median background signal intensities were subtracted from median signal intensities. A zero signal value was substituted for any negative signal intensities and all non-zero values were $\log _{2}$-transformed. In order to compensate for differences in expression levels between the two genomes represented on the array we analyzed each species separately, by normalizing across probes for each genome. For each species we imported $\log _{2}$-transformed data into SAS version 9 and used 'PROC MIXED' to model and remove systematic sources of variation contributed by Cy3 versus Cy5 dyes, slide and hybridization effects, slide $x$ dye interactions and slide $x$ treatment interactions $[36,37]$. Statistical residuals generated from the normalization step were subjected to mixed-model analyses of variance $\left(\log _{2}\right.$ normalized data for this experiment can be accessed at GEO Accession: GSE3742). We regarded as potentially meaningful any response that showed fold-change greater than four or that had $P$-values less than 0.0001 .

\section{Verification of expression with RT-qPCR}

We verified microarray results with real-time quantitative PCR from cDNA from both biological replicates used for microarray hybridization for four of the Buchnera genes that appeared to be upregulated under HS conditions (dnaK, ibpA, mopA, and yjeA), two Buchnera genes that did not show a HS response ( $\arg G$ and $c y s G$ ) and two Buchnera genes that appeared to be down-regulated under HS conditions (aroK and carA) as well as five aphid ESTs that appeared to be upregulated under HS conditions (EST5, EST6: hsp70, EST7, EST8 and EST10, Figure 1b), two aphid genes that did not show a heat shock response (EST13: ef $1 \alpha$ and EST14), and one aphid EST that appeared to be down-regulated under HS conditions (EST12, Figure 1b) following the protocol described in [38]. Primers were 
designed to amplify short regions of these genes and were as follows:

argG: F 5' TTCACTATGTCATGGTGCTACTG 3' and R 5' TGCCAGGAATTTTCATCTTTAC 3'; aroK: $\mathrm{F}$ 5' $^{\prime}$ CTGGGAAAAGCACTATTGGTC 3' and R 5' ACCTCCTCCTGTAGCAAGAAC 3'; carA: F 5' TTGCGATTACGCTATTCATGC 3' and R 5' CCTTAACAGGATGGTTGCCTC 3'; cysG: F 5' AGGTGGTGATCCCTTTATTTTC $3^{\prime}$ and R 5' GAGTATTTGCGATGTGTCAGTG 3'; dnaK: F 5' ATGGGTAAAATTATTGGTATTG $3^{\prime}$ and R 5' ATAGCTTGACGTTTAGCAGG 3'; ibpA: F 5' CCAATATCTGATACACCGAC 3' and R 5' TGAACAGATATATCTAATTCTTTTTC 3'; mopA: F 5' ATGTAAAAGACGGAAAAGG 3' and R 5' CCTGCTGGAGAAGAACTAG 3'; yjeA: F 5' CGAACAAAAAGGTTGATACCATTC 3' and R 5' TTATTAGCATCAGAAAGCGGATC 3'; EST13 - ef $1 \alpha$ : F 5 ' CTGATTGTGCCGTGCTTATTG $3{ }^{\prime}$ and R 5' TATGGTGGTTCAGTAGAGTCC 3'; EST6 - hsp70: F 5' TAAGAGGAAAACTAAAAAGGACG $3^{\prime}$ and $\mathrm{R} 5^{\prime}$ GGAAACTCGGGTGTAGAAATC 3'; EST14: F 5' TATGTTGCAGCAGCCGATAC 3 ' and R 5' AATGCTGGTCCTTCAGATGC 3'; EST5: F 5' CAAGAAATTCTCCCCTCATTTG 3' and R 5' TGCTCTTGCTAACCCACCAC 3'; EST7: F 5' TTCAAATCGTCGAAGTGTGC 3' and R 5' AAGTCGTCGACCGTGATTTC 3'; EST8: $\mathrm{F}$ 5' GCTGTTCTGCGTTCACTTGTAC $3^{\prime}$ and $\mathrm{R}$ 5' AGTCCGCTATTTTGGATATTGG 3 '; EST10 F 5 5' $^{\prime}$ TTTGGAAGGAATGGACTGTGG 3 ' and R 5 ' TTGAGCGTTGATTGTGTGGAC $\quad 3^{\prime} ; \quad$ EST12: F GAAAGAAGATTTGGTGCTTGG $3{ }^{\prime}$ and $\mathrm{R}$ 5' CTATTATGGGCACTTGCGATC 3'.

\section{Verification of spot identity with select resequencing across the array}

Spots that showed a $>4$ fold-change $\left(\log _{2}\right.$ fold-change $= \pm$ $2)$ and/or a significant fold-change $(\geq-\log (P)=5)$, together with the set of ESTs that we annotated through blast-searching as HS genes were resequenced using vector primer, $\mathrm{T} 3$, to confirm spot identity. The identity of the majority of resequenced spots (24/36 spots) was verified by a blastn search restricted to the NCBI "EST_other" database [39], which includes the EST sequences for the fragments on our array. A subset of resequenced spots (11/36 spots) returned poor sequence, as expected if more than one template was present. However, three of these, CN585306 (EST3, Buchnera mopA), CN586357 (aphid $d n a J)$ and CN586725 (EST6, aphid hsp70), were annotated as HS genes and showed large and significant upregulation under HS conditions. The correspondence between gene annotation and change in expression for these genes indicates that the expected gene is present on the array in the correct position. This observation indicates that most elements on the array contain the correct sequence.

\section{Investigation of cross-hybridization of heat-shock gene products}

We investigated cross-hybridization of the most upregulated heat shock genes on our array by spiking control RNA with labeled PCR products corresponding to HS genes. Four Buchnera genes (dnaK, ibpA, mopA and yjeA) and one aphid gene (hsp70) were amplified. PCR reactions were carried out for each gene in two $25 \mu \mathrm{l}$ volumes containing $50 \mathrm{mM} \mathrm{KCl}, 10 \mathrm{mM}$ Tris- $\mathrm{HCl}, 1.5 \mathrm{mM} \mathrm{Mg}^{2+}$, $2.5 \mathrm{mM}$ of each dATP, dCTP and dGTP, $1 \mathrm{mM}$ dTTP, 1.5 $\mathrm{mM}$ amino-allyl linked dUTP (Sigma A0410-1MG), 0.25 units of Eppendorf Taq DNA polymerase and $10 \mathrm{ng}$ of DNA (Buchnera genes) or cDNA (EST6 - hsp70). Primers used to amplify genes selected for spiking were as follows: Buchnera of A. pisum dnaK: F 5' CACCAGAGAGAACTCCTCCC 3' and R 5' ATGGATGGCAATAAACCACG 3', Buchnera of A. pisum ibpA: F 5' TCAATTAACACTAAGTATTCCTGG 3' and R 5' TAGGTTTTTCCTCTTCCGG 3', Buchnera of A. pisum mopA: $\mathrm{F}$ 5' ACCACAACAGCAACATTATTAGC $3{ }^{\prime}$ and R $5^{\prime}$ ACCTCCAGCAACTACACCTTC 3', Buchnera of A. pisum yjeA: F 5' GAAGCGTTTATTAGCATCAG $3^{\prime}$ and $\mathrm{R} 5^{\prime}$ TGAACAAGGAGGTAAGCC 3', A. pisum hsp70: F 5' GTGTTGATATTTGACCTGGGC 3 ' and R 5' AATGGTAGAGTTGCGTTCGAC 3 .

We performed a standard dye-flip hybridization experiment using RNA isolated from a control line and spiked with either $20 \mathrm{ng}$ of the aphid $h s p 70$ PCR product or $20 \mathrm{ng}$ of each of the four Buchnera gene PCR products. Spikes were added to the RNA samples following generation of cDNA and prior to cy3 and cy5 labelling. Thus, for one hybridization, the aphid spike was cy3 labeled and the Buchnera spikes were cy5 labeled and for the second hybridization the dyes were reversed. In this way we were able to distinguish between spots that were cross-hybridizing with aphid $h s p 70$ versus the Buchnera genes. Data from the spike array hybridization experiment were analyzed using the analysis pipeline described above for the heat shock experiments.

\section{List of abbreviations}

EST: expressed sequence tag

HS: heat shock

qPCR: quantitative-PCR

RT-qPCR: reverse-transcriptase quantitative-PCR

\section{Authors' contributions}

DLS provided CDNA libraries. WBH sequenced and provided clones of A. pisum ESTs. DLS and NAM designed the array. GKD PCR-amplified aphid ESTs. HED designed primers for and PCR-amplified Buchnera of A. pisum 
genes. ACCW and HED executed heat shock experiments and prepared RNA for array hybridization. ACCW annotated the array and analyzed the microarray data. HED designed, executed and analyzed RT-qPCR reactions. ACCW verified spot identity by resequencing. HED, ACCW and NAM designed and executed the spike experiment. ACCW and NAM drafted the manuscript. All authors read and approved the final manuscript.

\section{Acknowledgements}

P. Dang, Genomics Lab, USDA, ARS, U.S. Horticultural Research Lab, Ft. Pierce FL, P. Degnan and S. Miller provided technical assistance. R. Sprissler and K. Larsen of the Genomic Analysis and Technology Core, University of Arizona, provided technical assistance with microarray printing, hybridization and scanning. B. Nankivell assisted with figure preparation. This work was supported by National Science Foundation Genome-Enabled Biocomplexity grant \#03 I 3737 to NAM; National Institutes of Health (NIH) NRSA Fellowship \#5F32GM069 102-03 to GKD; NIH grant \#IROIGM63622-0I, Princeton University funds, and a David \& Lucile Packard Fellowship to DLS. ACCW was supported by NIH Training Grant \#I KI2 GM00708 to the Center for Insect Science, University of Arizona.

\section{References}

I. Buchner P: Endosymbiosis of Animals with Plant Microorganisms. New York, Interscience; 1965.

2. Moran NA, Munson MA, Baumann P, Ishikawa $\mathrm{H}$ : A molecular clock in endosymbiotic bacteria is calibrated using the insect hosts. Proc R Soc Lond B 1993, 253:167-171.

3. Baumann P, Baumann L, Lai CY, Rouhbakhsh D, Moran NA, Clark MA: Genetics, physiology, and evolutionary relationships of the genus Buchnera: Intracellular symbionts of aphids. Annual Review of Microbiology 1995, 49:55-94.

4. Douglas AE: Nutritional interactions in insect-microbial symbioses: Aphids and their symbiotic bacteria Buchnera. Annual Review of Entomology 1998, 43:17-37.

5. Shigenobu S, Watanabe H, Hattori M, Sakaki Y, Ishikawa H: Genome sequence of the endocellular bacterial symbiont of aphids Buchnera sp. APS. Nature 2000, 407:81-86.

6. Moran NA, Degnan PH: Functional genomics of Buchnera and the ecology of aphid hosts. Molecular Ecology in press.

7. Blackman RL, Eastop VF: Aphids on the world's crops: An identification and information guide. 2nd edition. Chichester, John Wiley \& Sons Ltd.; 2000:466.

8. Moran NA: The evolution of aphid lifecycles. Annu Rev Entomol 1992, 37:321-348.

9. Miura T, Braendle C, Shingleton A, Sisk G, Kambhampati S, Stern DL: A comparison of parthenogenetic and sexual embryogenesis of the pea aphid Acyrthosiphon pisum (Hemiptera: Aphidoidea). Journal of Experimental Zoology 2003, 295B:59-8I.

10. [http://www.genome.gov/10002/54]

II. Hunter WB, Dang PM, Bausher MG, Chaparro JX, McKendree W, Shatters Jr RG, McKenzie CL, Sinisterra XH: Aphid biology: Expressed genes from alate Toxoptera citricida, the brown citrus aphid. Journal of Insect Science 2003, 3:23-29.

12. Tagu D, Prunier-Leterme N, Legeai F, Gauthier JP, Duclert A, Sabater-Muñoz B, Bonhomme J, Simon JC: Annotated expressed sequence tags for studies of the regulation of reproductive modes in aphids. Insect Biochemistry and Molecular Biology 2004, 34:809-822.

13. Nakabachi A, Shigenobu S, Sakazume N, Shiraki T, Hayashizaki Y, Carninci $P$, Ishikawa $H$, Kudo T, Fukatsu T: Transcriptome analysis of the aphids bacteriocyte the symbiotic host cell that harbors an endocellular mutulaistic bacterium, Buchnera. Proceedings of the National Academy of Sciences of the United States of America 2005, 1 02:5477-5482.

14. Shoemaker DD, Schadt EE, Armour CD, He YD, Garrett-Engele P, McDonagh PD, Loerch PM, Leonardson A, Lum PY, Cavet G, Wu LF, Altschuler SJ, Edwards S, King J, Tsang JS, Schimmack G, Schelter JM, Koch J, Ziman M, Marton MJ, Li B, Cundiff P, Ward T, Castle J,
Krolewski M, Meyer MR, Mao M, Burchard J, Kidd MJ, Dai H, Phillips JW, Linsley PS, Stoughton R, Scherer S, Boguski MS: Experimental annotation of the human genome using microarray technology. Nature 2001, 409:922-927.

15. Gaasterland T, Oprea M: Whole-genome analysis: annotations and updates. Current Opinion in Structural Biology 200I, I I:377-38I.

16. van Ham RCHJ, Kamerbeek J, Palacios C, Rausell C, Abascal F, BastoIla U, Fernandez JM, Jimenez L, Postigo M, Silva FJ, Tamames J, Viguera E, Latorre A, Valencia A, Moran F, Moya A: Reductive genome evolution in Buchnera aphidicola. PNAS 2003, 100:58I-586.

17. Tamas I, Klasson L, Canback B, Naslund AK, Eriksson AS, Wernegreen JJ, Sandstrom JP, Moran NA, Andersson SGE: 50 Million Years of Genomic Stasis in Endosymbiotic Bacteria. Science 2002, 296:2376-2379.

18. Moran NA, Wernegreen J]: Lifestyle evolution in symbiotic bacteria: insights from genomics. Trends in Ecology and Evolution 2000, I 5:32I-326.

19. Ochman H, Moran NA: Genes lost and genes found: Evolution of bacterial pathogenesis and symbiosis. Science 200I, 292:1096-1098.

20. Handelsman J: Metagenomics or Megagenomics? Nature Reviews Microbiology 2005, 3:457-458.

21. Barnett MJ, Toman C], Fisher RF, Long SR: A dual-genome Symbiosis Chip for coordinate study of signal exchange and development in a prokaryote-host interaction. Proceedings of the National Academy of Sciences of the United States of America 2004, I0I:|6636-|664|.

22. Wilcox JL, Dunbar HE, Wolfinger RD, Moran NA: Consequences of reductive evolution for gene expression in an obligate endosymbiont. Molecular Microbiology 2003, 48: |49|-1500.

23. Feder ME, Hofmann GE: Heat-shock proteins, molecular chaperones, and the stress response: Evolutionary and Ecological Physiology. Annual Review of Physiology 1999, 6 1:243-282.

24. Asin L, Pons X: Effect of high temperature on the growth and reproduction of corn aphids (Homoptera: Aphididae) and implications for their population dynamics on the northeastern Iberian peninsula. Environmental Entomology 2001, 30: I I27-I I34.

25. Ma CS, Hau B, Poehling HM: The effects of heat stress on the survival of the rose grain aphid, Metopolophium dirhodum (Hemiptera: Aphididae). European Journal of Entomology 2004, | 0 l:327-331.

26. Turak E, Talent R, Sunnucks $P$, Hales DF: Different responses to temperature in three closely-related parthenogenetic cereal aphids. Entomologia Experimentalis et Applicata 1998, 86:49-58.

27. Wang JJ, Tsai JH: Effect of temperature on the biology of Aphis spiraecola (Hompotera: Aphididae). Annals of the Entomological Society of America 2000, 93:874-883.

28. Ohtaka C, Ishikawa H: Effects of heat treatment on the symbiotic system of an aphid mycetocyte. Symbiosis 1991, I I:19-30.

29. Montllor CB, Maxmen A, Purcell AH: Facultative bacterial endosymbionts benefit pea aphids Acyrthosiphon pisum under heat stress. Ecological Entomology 2000, 27:189-195.

30. Moreau Y, Aerts S, De Moor B, De Strooper B, Dabrowski M: Comparison and meta-analysis of microarray data: from the bench to the computer desk. Trends in Genetics 2003, 19:570-577.

31. Xu W, Bak S, Decker A, Paquette S, Feyereisen R, Galbraith DW: Microarray-based analysis of gene expression in very large gene families: the cytochrome $\mathbf{P 4 5 0}$ gene superfamily of Arabidopsis thaliana. Gene 2001, 272:61-74.

32. Komoto N, Sezutsu H, Yukuhiro K, Banno Y, Fujii H: Mutations of the silkworm molybdenum cofactor sulfurase gene, og, cause translucent larval skin. Insect Biochemistry and Molecular Biology 2003, 33:4I 7-427.

33. McGraw EA, O'Neill SL: Wolbachia pipientis: intracellular infection and pathogenesis in Drosophila. Current Opinion in Microbiology 2004, 7:67-70.

34. Sabater-Muñoz B, Legeai F, Rispe C, Bonhomme J, Dearden P, Dossat C, Duclert A, Gauthier JP, Giblot Ducray D, Hunter W, Dang P, Kambhampati S, Martinez-Torres D, Cortes T, Moya A, Nakabachi A, Philippe C, Prunier-Leterme N, Rahbé Y, Simon JC, Stern D, Wincker $P$, Tagu D: Large-scale gene discovery in the pea aphid Acyrthosiphon pisum (Hemiptera). Genome Biology in press.

35. Moran NA, Russell JA, Koga R, Fukatsu T: Evolutionary relationships of three new species of Emterobacteriaceae living as 
symbionts of aphids and other insects. Applied and Environmental Microbiology 2005, 7 I:3302-33I0.

36. Wolfinger RD, Gibson G, Wolfinger ED, Bennett L, Hamadeh $H$ Bushel P, Afshari C, Paules RS: Assessing gene expression significance from cDNA microarray expression data via mixed models. Journal of Computational Biology 200I, 8:625-637.

37. Gibson G, Wolfinger RD: Gene expression profiling using mixed models. In Genetic analysis of complex traits using SAS Edited by: Saxton AM. Cary, N.C., SAS Users Press; 2004:25I-279.

38. Moran NA, Dunbar HE, Wilcox JL: Regulation of Transcription in a Reduced Bacterial Genome: Nutrient-Provisioning Genes of the Obligate Symbiont Buchnera aphidicola. I Bacteriol 2005, 187:4229-4237.

39. NCBI: . [http://www.ncbi.nlm.nih.gov/BLAST/].

Publish with Biomed Central and every scientist can read your work free of charge

"BioMed Central will be the most significant development for disseminating the results of biomedical research in our lifetime. "

Sir Paul Nurse, Cancer Research UK

Your research papers will be:

- available free of charge to the entire biomedical community

- peer reviewed and published immediately upon acceptance

- cited in PubMed and archived on PubMed Central

- yours - you keep the copyright

Submit your manuscript here:

http://www.biomedcentral.com/info/publishing_adv.asp
BiolMedcentral 